\title{
Understanding Strain And Irradiation Segregation In Fusion Materials
}

Andrew London ${ }^{1}$, Chris Hardie ${ }^{1}$, Tonci Tadić ${ }^{2}$, Suchandrima Das ${ }^{3}$, Felix Hofmann ${ }^{3}$ and Stjepko Fazinić ${ }^{2}$

${ }^{1}$ UKAEA, Abingdon, United Kingdom, ${ }^{2}$ Ruđer Bošković Institute, Zagreb, Croatia, ${ }^{3}$ University of Oxford, Oxford, United Kingdom

In a fusion power plant, both structural and armour materials experience irradiation over a wide range of temperatures. Therefore, there is a need evaluate and screen materials for sensitivities to irradiation temperature. Experimental validation of the mechanisms of irradiation damage is also required with high temperature fidelity. Fusion neutron sources are lacking, however ion irradiation can quickly reproduce most features of the damage caused by high-energy neutrons. Using samples exposed using a novel ion irradiation technique which provides a wide range of irradiation temperatures [1], we show how nanoindentation can reveal the mechanical response as a function of irradiation temperature and the role microstructure plays in this response in $\mathrm{Fe}-\mathrm{Cr}$ alloys.

Reduced-activation ferritic steels are a primary candidate for structural components due to high strength at elevated temperatures, low induced radioactivity, improved swelling resistance over stainless steels and comparatively low cost. Investigating how the atomic scale defects caused by neutron bombardment agglomerate and evolve with time and temperature is critical to understand their influence on dislocation plasticity and resulting macroscopic mechanical properties.

Several high-purity $\mathrm{Fe}-\mathrm{Cr}$ binary alloys were irradiated with a range of irradiation temperatures between 150 and $450{ }^{\circ} \mathrm{C}$ to $0.2 \mathrm{dpa}$ at the RBI's DiFU dual-beam ion irradiation facility in Croatia. Nanoindentation was used to investigate the irradiation hardening response as a function of irradiation temperature and the results informed later site-specific microscopy. The microstructure of the irradiated material was examined by Transmission Electron Microscopy (TEM) and Atom Probe Tomography (APT). TEM revealed the size and density of dislocation loops produced during the irradiation and APT showed the level of irradiation induced segregation of $\mathrm{Cr}$ and interstitial impurities.

To further improve the analysis of the APT data, a morphological analysis of the iso-concentration surfaces representing the nanoscale microstructural features was made [2]. Using methods developed by the astrophysics community to describe the shape of superclusters of galaxies, we described second-phase regions using Minkowski functionals, representing the regions' volume, surface area, mean curvature and Euler characteristic. This method mathematically quantifies these morphological measurements without the need for user-tuneable parameters.

Finally, to explore the irradiated microstructure non-destructively, X-ray micro-Laue diffraction was performed [3]. By this method we determined the out of plane lattice strains caused by self-ion implantation damage, and their dependence on irradiation temperature. The measured strain and its distribution gives insight to the arrangement of irradiation defects which is critical for our understanding of the evolution of these microstructures. This has important implications for the lifetime of components in a nuclear environment and how these effects may be mitigated by alloy design or annealing.

This work has been carried out within the framework of the EUROfusion Consortium and has received funding from the Euratom research and training programme 2014-2018 and 2019-2020 under grant agreement No 633053. The views and opinions expressed herein do not necessarily reflect those of the European Commission. The work was supported by EPSRC Grant is EP/T012250/1. The research used UKAEA's Materials Research Facility, which has been funded by and is part of the UK's National Nuclear User Facility and Henry Royce Institute for Advanced Materials. This research used resources of the Advanced Photon Source, a U.S. 
Department of Energy (DOE) Office of Science User Facility operated for the DOE Office of Science by Argonne National Laboratory under Contract No. DE-AC02-06CH11357.

\section{References}

[1] CD Hardie et al., Exploitation of thermal gradients for investigation of irradiation temperature effects with charged particles, Scientific Reports 9 (1), 1-10, 2019

[2] DR Mason, AJ London, Morphological analysis of $3 \mathrm{~d}$ atom probe data using Minkowski functionals, Ultramicroscopy 211, 112940, 2020

[3] S Das et al., The effect of helium implantation on the deformation behaviour of tungsten: X-ray microdiffraction and nanoindentation, Scripta Materialia 146, 335-339, 2018 